\title{
Novel based Approach Schema for Processing Vehicle Tracking System through GPS SMS Gateways
}

\author{
Sermakani. A. M1, Veena. T², Julia Faith. $S^{2}$ \\ ${ }^{1}$ Associate Professor, ${ }^{2}$ Assistant Professor \\ 1,2Department of IT, S. A. Engineering College, Chennai, Tamil Nadu, India
}

\begin{abstract}
In recent years many systems are approached to track the vehicles in order to find out movement of any equipped vehicle from any location at any time. This paper implements formalised schema to develop a tracking system that is much cost effective that available in the market. Various technologies utilised are GPS (Global Positioning System) module to get geographic coordinates at regular time intervals. The GSM/GPRS (Global System Positioning/General Packet Radio Service) module is used to transmit and update the vehicle location to a database. The Google Maps API is used to display the vehicle position on the map in the Smartphone application. The tracking system here helps to identify the location of the college bus through our smart phone when a SMS (Short Message Service) is sent to a personal number thus indicating the bus location via SMS. By incorporating a GPS and GSM modem the location of the device by sending a SMS to the number specified. No external server or internet connection is used in knowing the location at user end which in return reduces the cost. We can also use this technology to reduce our time whenever we are waiting for buses by knowing their location on our mobile phones. If vehicle has been stolen then also we can track our vehicles.
\end{abstract}

Keywords: GPS, GSM/GPRS, Google Maps API, Student alert message

\section{INTRODUCTION}

A vehicle tracking system is an electronic device installed in a vehicle to enable the user to track the vehicle location. A GPS-based vehicle tracking system will inform where your vehicle is and where it has been, how long it has been. GPS and GSM based vehicle location and tracking system will provide effective, real time vehicle location, mapping and reporting this information back to monitoring device and improving the level of service provided ${ }^{[1]}$.GPS receiver used to send the position (Latitude and Longitude) of the vehicle from a remote place. The system uses geographic position and time information from the Global Positioning Satellites. The basic function of the device in the bus is to acquire and transmit the position of the bus to the server at a fixed interval of time. Microcontroller unit forms the heart of the tracking unit, which acquires and process the position data from the GPS module. The communication interface is responsible for receiving the signals from the satellites and sending the information to the server. If the vehicle is stolen, the location data from tracking system can be used to find the location and can be informed to police for further action. The Google Maps API is used to display the current location of the vehicle on the map in the Smartphone application. The principal goal of the project was to build a GPS vehicle tracking device to provide a more user-friendly, affordable, and an efficient means to track vehicles accurately ${ }^{[2]}$. When functioning correctly, the device uses a GPS device to track the current location. This is very helpful for schools, colleges, logistic companies, heavy vehicle owners in managing their time and to increase the security of the vehicle by monitoring and tracking the locations. This will find the speed of the vehicle in real time to create a user friendly transportation system using GSM. This will send information about the location of buses to the parents via SMS. The waiting time for students and parents who are waiting in the bus stop was reduced. To offer cost effective mobile information service to user via SMS [3].
Initially the GPS will give the current Latitude and Longitude value. The Co-ordinates (Latitude and Longitude) which is received is transferred from the GPS receiver to the server. It uses internet for the data transfer between receiver and server. The microcontroller checks for a closest location match inside the server with the received GPS coordinate data. The matched location detail is sent to the SMS providers for the further process ${ }^{[4]}$.The alert message is send to the students who are about to take the bus at next stop thus it saves the time of students.

Google Map is a version of Google Earth that shows the maps on-line using with a web server and a web browser. The satellite locations are monitored and stored in the server. The server on request from the users will show the current location of the vehicle on the Google Map. In case of any emergency the alert messages can be sent to the nearby hospitals/police stations. The college is informed through the SMS that the vehicle is in trouble.

\section{LITERATURE SURVEY}

R. Maruthi, C. Jayakumari. et.al has developed the system has "SMS based Bus Tracking System using Open Source Technologies" This system is to develop a system that manages and controls the transport using a tracking device to know the scheduled vehicle and the current location of the vehicle via SMS using a GPS tracking device.

Kunal Maurya. has developed the system has" Real Time Vehicle Tracking System using GSM and GPS Technology-An Anti-theft Tracking System "This sytem is used for tracking and positioning of any vehicle by using Global Positioning System (GPS) and Global system for mobile communication $(\mathrm{GSM})^{[5]}$. This vehicle tracking system can be found in consumers vehicles as a theft prevention and retrieval device seokJu Lee. has developed the system has" Design and implementation of vehicle tracking system using GPS/GPRS 
Technology and smart phone application "The Google maps API is used to display the vehicle on the map in the smart phone application[6]

\section{ARCHITECTURE MODEL:}

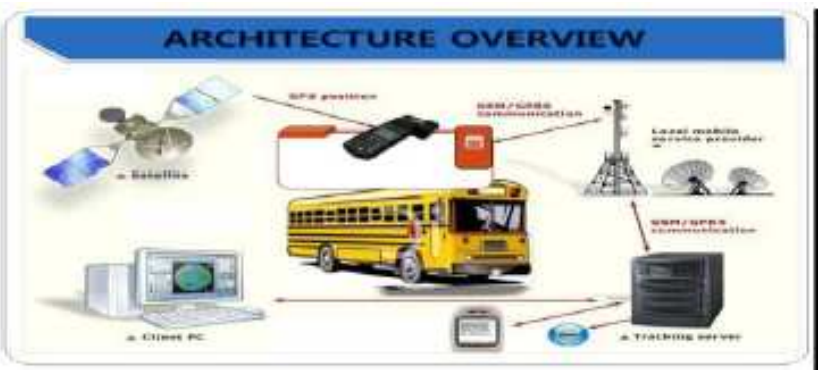

Fig 1: Architectural model

\section{PROPOSED SYSTEM}

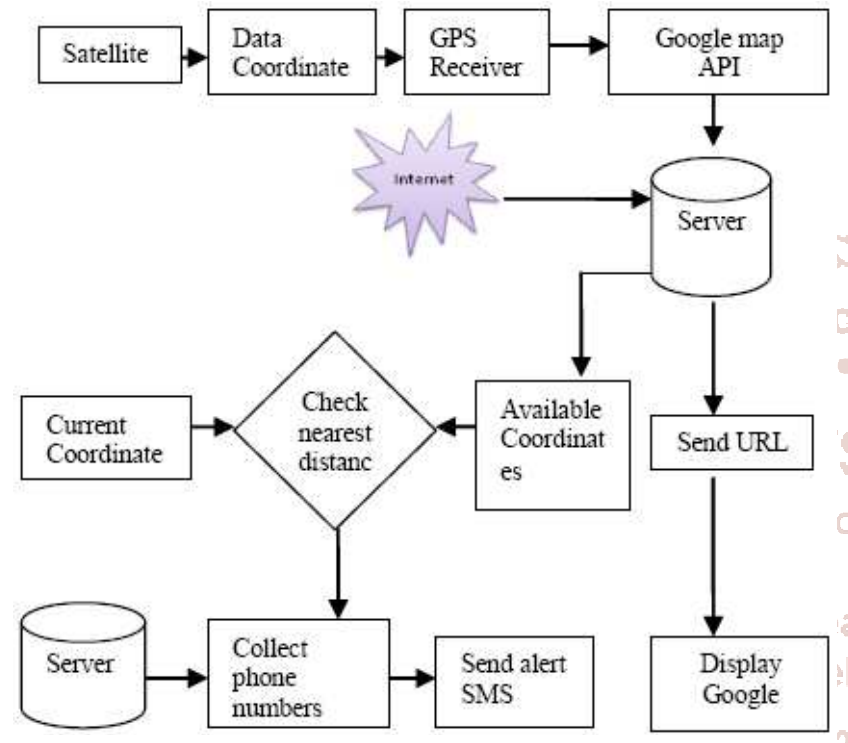

Fig 2: Detailed Design

The proposed System is to track and monitor the vehicle location using GPS, GPRS and GSM modules. The GPS will send the data to the server and calculate the distance between two points for a fixed time interval. This system will establish a communication between server and bus system When the vehicle is approaching near the server will Send an alert messages to the students. It is capable to provide a realtime data regarding the current location of buses through Google Map. In case of any emergency this will Send an alert messages to college. Tracking the nearby hospitals and police stations in case of any emergency. Reduced usage of data used for tracking.

\section{IMPLEMENTATION}

The implementation of the system contains various modules. The Global Positioning System (GPS) is a space-based satellite navigation system that provides location and time information in all weather conditions, anywhere on or near the Earth where there is an unobstructed line of sight to four or more GPS satellites. It is maintained by the United States government and is freely accessible to anyone ${ }^{[7]}$.This is used to give the Data coordinates value (latitude ,longitude).The coordinates(Latitude and Longitude) received is transferred from the GPS receiver to the server. There is a Communication betweeen the GPS receiver and server. It uses internet for the data transfer between receiver and server. The microcontroller is act as a heart of the project.
The microcontroller checks for a closest location match inside the server with the received GPS coordinate data. The Condition will check for a fixed amount of time. If the condition was satisfied then the module will collect the phone numbers from the server. The phone number which is collected is send to SMS . Once the SMS provider received the phone number. The Alert messages are sent to the students who are about to take the bus at next stop thus it saves the time of students. Google Map is a version of Google Earth that shows the maps on-line using with a web server and a web browser [8]. The satellite locations are monitored and stored in the server. The GPS receiver gives request to Google map. The Google map request to the server.The server will send an url to the user .By clicking the url the user can view the location of the vehicle through google map. The server on request from the users will show the current location of the vehicle on the Google Map. In case of any emergency the alert messages can be sent to the nearby hospitals/police stations. The college is informed through the SMS that the vehicle is in trouble. By this application we know where your vehicles are at all times $-24 * 7$ in realtime. Monitor your vehicles covertly. View your real-time tracking device location from your computer or phone. Monitor speed and safe driving habits of your crew. Receive notifications of driver [9].Cost effective and secured

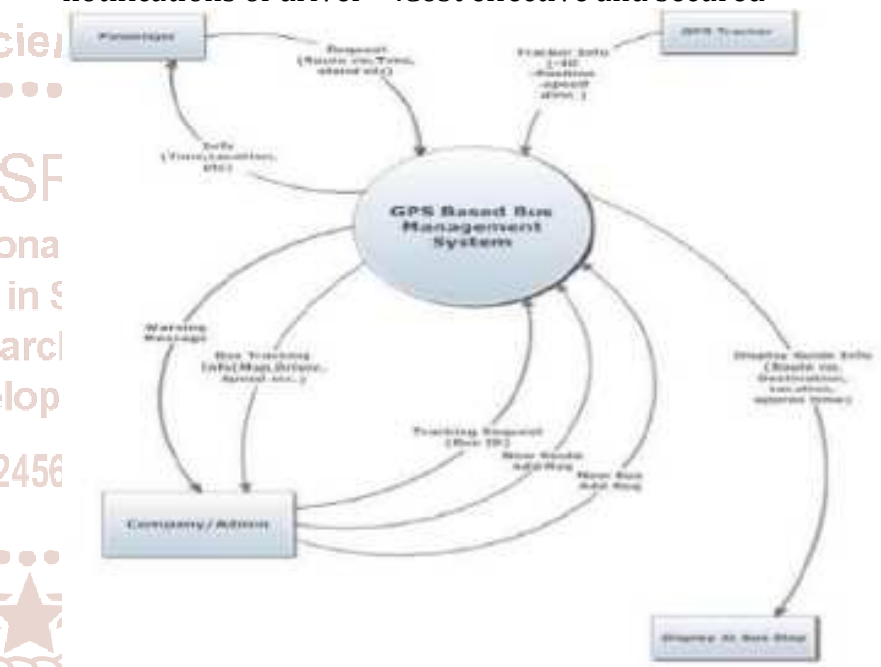

Fig 3: Data Flow Diagram

\section{A. Admin Control Module}

Admin Enter the Admin name and Password to login. Admin register the students by entering the details of the students. Admin register the devices by entering the details of the bus. Update the database. Admin receives alert in case of emergency. Admin can view the current status and history. Admin contacts college in case of any emergency. In this module the admin can view the status of the message arrival. The admin can view and update the datas in the database. In case of any emergency the alert message is send to the college.

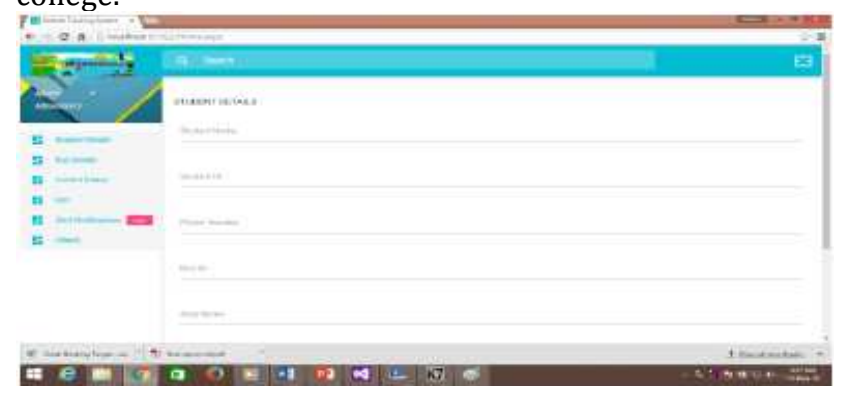

Fig 4: Storing details 


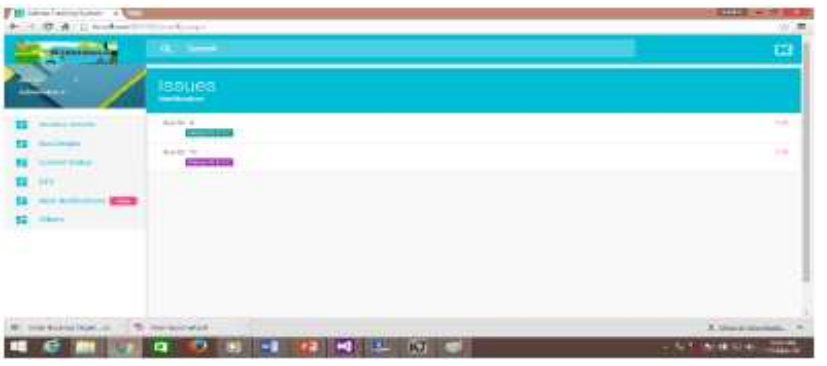

Fig 5: Showing Alert Notification

\section{B. GPS Module:}

The Global Positioning System (GPS) is a space-based satellite navigation system that provides location and time information in all weather conditions, anywhere on or near the Earth where there is an unobstructed line of sight to four or more GPS satellites. It is maintained by the United States government and is freely accessible to anyone A GPS-based vehicle tracking system will inform where your vehicle is and where it has been, how long it has been GPS devices works in all weather conditions, anywhere on or near the Earth where there is an unobstructed line of sight to four or more GPS satellites. A GPS receiver listens to multiple satellites and solves equations to determine the exact position of the receiver and its deviation from true time.

\section{GSM and GPRS Module}

General Packet Radio Service GPRS is a packet switched service based on Global System for Mobile Communications GSM. A GSM modem is a wireless modem that works with a GSM wireless network. A wireless modem behaves like a dial-up modem. The main difference between them is that a dial-up modem sends and receives data through a fixed telephone line while a wireless modem sends and receives data through radio waves The co-ordinates (Latitude and Longitude) received is transferred from the GPS receiver to the server. It uses internet for the data transfer between receiver and server. GPRS was used for data transfer instead of SMS. The data are transferred as a packet to the server. And the server acknowledges after receiving the packets.

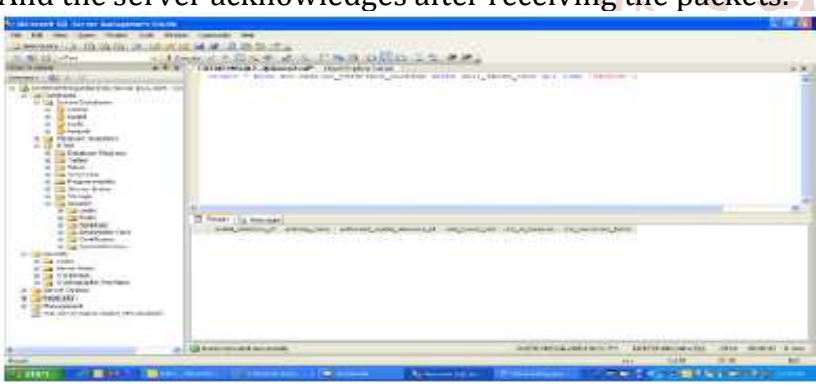

Fig 6: Storage of Latitude and Longitude

\section{Processing Module:}

This module is the core heart of the project. The microcontroller checks for a closest location match inside the server with the received GPS coordinate data. The matched location detail is sent to the SMS providers for the further process.

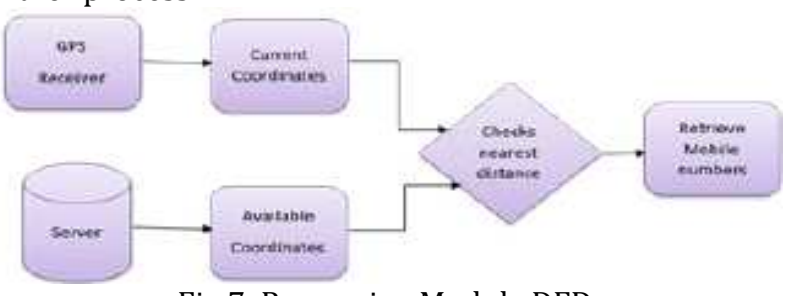

Fig 7: Processing Module DFD

\section{E. SMS Alert module}

Alert messages are sent to the students. Students receives alerts when the vehicle reach near the point. It saves the waiting time of the student. The alert message is sent through the SMS providers. The students who are near to the location of the device are found using the algorithm. The phone numbers of the students are identified and the details are sent to the SMS providers

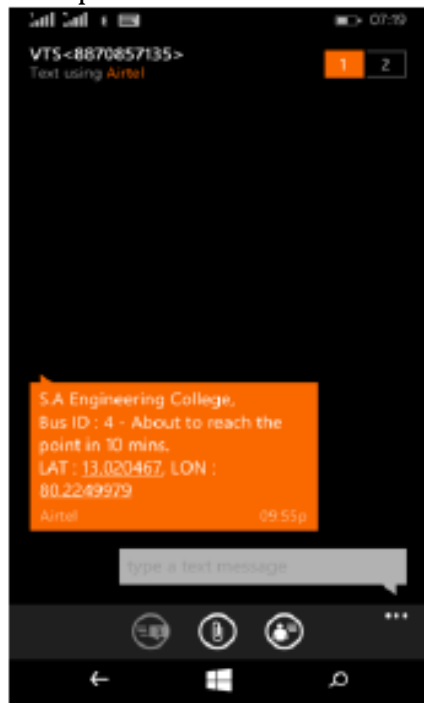

Fig8: Sending alert message to the user

\section{F. Google Map}

Google Map is a version of Google Earth which shows the maps on-line using with a web server and a web browser. This module is used to show the location of the bus via Google Map on the smart phone application in real time using HTTP request. suppose if the student missed the bus, the students can able to track the location of the bus in google map. The google map API automatically handles access to the Google map server, display map and responds to user.

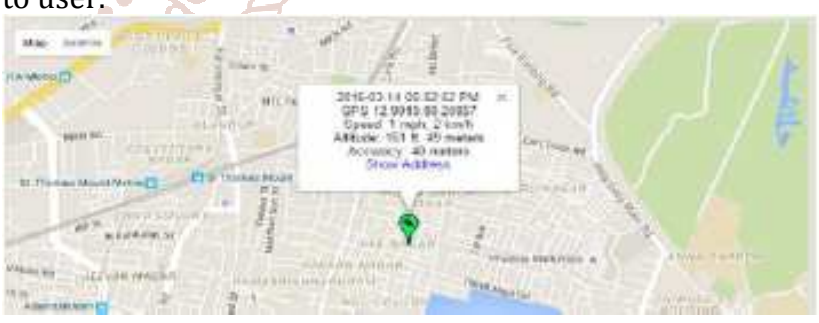

Fig 9 Current Position on the Google Map

\section{ALGORITHM}

The Algorithm which is used is shortest path technique.This solves the single source shortest path problem. It is applied only on positive weights graphs ${ }^{[10]}$. This algorithm is often used in routing.

The shortest distance between two given points $\mathrm{P}_{1}=\left(\mathrm{lat}_{1}\right.$, $\left.\operatorname{lon}_{1}\right)$ and $\mathrm{P}_{2}=\left(\mathrm{lat}_{2}, \mathrm{lon}_{2}\right)$ on the surface of a sphere with radius $R$ is the circle distance. It can be calculated using the formula:

Dist $=\arccos \left(\sin \left(\mathrm{lat}_{1}\right) \cdot \sin \left(\mathrm{lat}_{2}\right)+\cos \left(\mathrm{lat}_{1}\right) \cdot \cos \left(\mathrm{lat}_{2}\right) \cdot \cos \right.$ $\left.\left(\operatorname{lon}_{1}-\operatorname{lon}_{2}\right)\right) \cdot \mathrm{R}$

By using the position we calculate the distance from source to every node in the graph. From this we also estimate the shortest path. Distance is given by a formula: 
Distance $=\left[(\mathrm{x} 2-\mathrm{x} 1)^{2}+(\mathrm{y} 2-\mathrm{y} 1)^{2}+(\mathrm{z} 2-\mathrm{z} 1)^{2}\right]^{1 / 2}$

Where $\mathrm{x}, \mathrm{y}, \mathrm{z}$ are the coordinates of a position given by GPS.

\section{Pseudo code:}

Step 1 : Identify Source S as Permanent \& All other nodes V.D $[\mathrm{s}]=0 \& \mathrm{D}[\mathrm{v}]=\infty$

Step 2 : Turn On GPS \& Get current position For Source node in form of coordinates and calculate distance.

Step 3 : Set V as Temporary and update neighbours state

Step 4 : If the temporary Node linked to $S$ that has loweest weight. Get position of that node by GPS and calculate Distance

Step 5 : check whether the node is destination or not if no, follow step 3.if yes means

Step 6 : The information is send to the user mobile

VI. PEFORMANCE MEASURE AND OPTIMIZATION

The process of map would show a single bus on each route and the user had no control over which bus was shown. In Proto type two, the user could see all the buses on any given route as well as the information specific to each bus ${ }^{[11]}$. The text on the real time tracking page before was quite lengthy and may have distracted users from the pages main purpose. In prototype two, the page was made much shorter by moving the SMS texting instructions to its own page and providing a link for users to navigate to the page if they were looking for that information. The intent of the real time tracking page is much clearer now as the route selection interface is very prominent on the page and the use of bright hover states draws the user's attention [12].

\section{CONCLUSION}

The novel vehicle tracking system uses the wireless communication technique and was successfully designed and tested for real time data [13]. The system has the advantages of small size, low costs, full-featured and powerful expansibility.

It can be easily installed and used in the buses to ease the burden of transport department as the educational institutions have large number of buses. This system is based on embedded system and can also be developed on android platform [14][15]. This system proved to be much more efficient and produced good results such as, Sending location of bus to college in every 2 minutes. In case of accident sends Alert to college. Send alerts message to the student before the bus arrives their stop. Also Addition/update/deletion of student database is easy.

\section{FUTURE SCOPE}

In future the proposed system can be enhanced by collecting the traffic details of the route by which the vehicle is travelling. If there seems like heavy traffic the alert signal is given to the driver, so that the driver can choose alternate way. Thus it saves the travelling time. Also in case of any emergency the alert messages can be sent to police stations, hospitals for the quick recovery. In case of bus failure driver can select the problem and inform college and contact the Mechanic automatically

\section{REFERENCES}

[1] N. Vijayalashmy, V. Yamuna, G. Rupavani, A. Kannaki @ Vasan tha Azhagu, "GNSS based bus monitoring and sending SMS to the passengers," International Journal of Computer Applications (C) 2015 by IJCA Journal Volume 119 - Number 10.

[2] Amol Dhumal, Amol Naikoji, Yutika Patwa" Vehicle Tracking System using GPS and Android OS" International Journal of Advanced Research in Computer Engineering \& Technology (IJARCET) Volume 4 Issue 4, April 2015

[3] Pradip Suresh Mane, Vaishali Khairnar, "Analysis of bus tracking system using GPS on smart phones," IOSR Journal of Computer Engineering (IOSR-JCE). e- ISSN: 2278-0661, p- ISSN: 2278-8727Volume 16, Issue 2, Ver. XII (Mar-Apr. 2014).

[4] R. Maruthi, C. Jayakumari, "SMS based bus tracking system using open source technologies," International Journal of Computer Application, Volume 86, No 9, January 2014.

[5] Kunal Maurya, Mandeep Singh, Neelu Jain "Real Time Vehicle Tracking System using GSM and GPS technology ".International Journal of Electronics and Computer Science Engineering ,ISSN- 2277-1956,april 2013.

[6] Eddie Chi-wah Lau,"simple Bus tracking system", Journal of Advanced Computer Science and Technology Research, vol3, No.1.2013.

[7] R. Ramani, S. Valarmathy, "vehicle tracking and locking system based on GSM and GPS", I. J. Intelligent Systems and Applications,2013,09,86.

[8] Abid Khan \& Ravi Mishra, -GPS - GSM Based Tracking System\|, International Journal of Trends and Technology, ISSN: 2231 - 5381, Volume 3, Issue 2, 2012.

[9] Montaster N. Ramadan Mohammad A. Al-Khedher, and Sharaf A. Al-Kheder, "Intelligent Anti-theft and tracking Stystem for automobiles", International Journal of machine learning and computing vol.2 No.1,February 2012.

[10] seokJu Lee-"Design and implementation of vehicle tracking system using GPS/GPRS Technology and smart phone application" Volume 1, Issue 2, 2012.

[11] J. Paek, J. Kim, and R. Govindan, "Energy-efficient rateadaptive gps-based positioning for smart phones," in MobiSys. ACM, 2010, pp. 299-314.

[12] K. Lin, A. Kansal, D. Lymberopoulos, and F. Zhao, "Energy accuracy aware localization for mobile devices," in MobiSys. ACM, 2010.

[13] Z. Zhuang, K.-H. Kim, and J. P. Singh, "Improving energy efficiency of location sensing on smart phones," in MobiSys. ACM, 2010, pp. 315330.

[14] Thiagarajan, L. Ravindranath, K. LaCurts, S. Madden, H. Balakrishnan, S. Toledo, and J. Eriksson, "Vtrack: accurate, energy aware road traffic delay estimation using mobile phones," in SenSys. ACM, 2009, pp. 85-98.

[15] M. B. Kjargaard, J. Langdal, T. Godsk, and T. Toftkjær, "Entracked: energy-efficient robust position tracking for mobile devices," in MobiSys. ACM, 2009, pp. 221234. 